\title{
VARIAZIONI DI TORSIONE PRODOTTE NEL FERRO E NEL NICAEL da UN CAMPO MaGNETICO ALTERNATIVO.
}

\author{
G. ERCOLINI.
}

È nota l'influenza della magnetizzazione sulla torsione del ferro e del nichel specialmente per gli studi del Prof. Cantone ${ }^{5}$ ) il quale, rilevando pel primo che le forze interne destate dalla magnetizzazione non sono affatto trascurabili, giunse a stabilire la legge che per ogni valore dell'intensità magnetica la variazione d'energia relativa all' effetto magnetico è compensata da quella corrispondente all' effetto elastico.

Io ho trovato che anche un campo magnetico alternativo longitudinale varia considerevolmente la torsione di fili di ferro e di nichel e qui espongo $i$ risultati a cui son giunto.

La disposizione sperimentale è la seguente: Ad un cilindro di ottone, raccomandato superiormente ad una mensola, $\dot{\theta}$ fissato il filo in esame, sempre della lunghezza di cm. 25, dalla eui estremità inferiore parte un cilindro di ottone, del diametro di cm. 0,2 e lungo cm. 20 , da cui pende un filo-dinamometro di ottone crudo del diametro di $\mathrm{cm} .0,05$ e della lunghezza di cm. 50, saldato inferiormente ad una sbarra metallica.

Allo scopo di produrre torsioni sotto carico costante questa sbarra porta, fissi alle estremità, due aghi da cucire che passano liberamente in due forellini praticati agli estremi d'un diametro d' un anello metallico girevole su di un altro sorretto da una mensola in modo che il suo centro si trova sul prolungamento del sistema sovrastante dei due fili. Le torsioni si producono rotando l' anello mobile di angoli che

1) Rendiconti del R. I. Lombardo, 1904. 
si leggono su di un disco graduato. I pesi tensori si attaccano ad un filo appeso alla metà della sbarra cogli aghi e passante, pel centro degli anelli, al di sotto della mensola che li sostiene.

Il filo in esame si trova sull' asse comune e verticale di due spirali, lunghe $35 \mathrm{~cm}$., di filo isolato avvolto su due tubi di differente diametro fra $i$ quali passa una corrente d' acqua fredda.

Per la misura delle torsioni subite dal filo in prova serve un lungo ed esilissimo filo di vetro che, collegato coll'estremità inferiore del sottile cilindro di ottone girevole interposto tra i due fili, scorre sopra un cerchio graduato su specchio. Ed uno smorzatore ad olio estingue le piccole vibrazioni del sistema causate da quelle, inevitabili, del suolo.

Le variazioni della torsione dei fili si misurano col metodo del cannocchiale e scala. Questa è fissata ad una parete a circa $400 \mathrm{~cm}$. dal filo ed è piegata in areo di cerchio; quello invece si trova a $100 \mathrm{~cm}$. dallo specchio che è solidale col cilindro di ottone girevole soltanto dopo che è stata prodotta la torsione voluta.

A questo scopo lo specchio stesso è portato da un leggero telaietto di legno, di forma opportuna, che può essere sollevato ed isolato da tutto il sistema dei due fili mediante l'innalzamento di una leva, spostabile esattamente solo in un piano verticale e raccomandata ad un adatto sostegno. Abbassando questa leva, mediante il rilasciamento d' un filo scorrevole in una carrucola, il telaio, senza la più lieve scossa, viene posato del tutto su di un diseo di sughero solidale col cilindro di ottone girevole. L'adesione è tale che lo specchio può seguire rapide ed assai ampie vibrazioni dei fili senza spostarsi sensibilmente.

Ciò posto il procedimento delle esperienze è semplice: sollevato lo specchio si genera la torsione voluta del filo in prova, si abbassa poi quello e, quando ve n'è bisogno, si aggiusta il cannocchiale; lanciata allora la corrente alternata (42 periodi) in una spirale, si osserva la variazione che si genera nella torsione del filo. 
Ferro.

I fili di ferro che ho adoprato sono tutti del diametro di cm. 0,04 e vengon ricotti nell' aria rarefatta con una corrente di 8 a. mantenuta costante per $180 \mathrm{~s}$, ma gradatamente crescente da prima e decrescente da ultimo.

Collocati a posto, vengon sempre in principio assoggettati a più cicli elastici di torsione bilaterale simmetrica.

Prima di riferire $\mathrm{i}$ risultati farò notare che allorquando agisce il campo alternato su di un filo torto, questo accusa delle vibrazioni torsionali che rendono un po' torbida l'immagine della scala nel cannocchiale. In generale però le letture possono farsi abbastanza bene, ma nelle grandi torsioni la vibrazione si fa - in generale e dipendentemente dal carico tensore e dall' intensità del campo - assai ampia, in modo che bisogna valutare ad occhio la posizione occupata dal reticolo fra una grande divisione e l' altra della scala o usando magari l'artifizio di fare scorrere un filo nero sulla scala stessa. Con un po' d' esercizio si riesce a fare le letture con assai speditezza e precisione.

Riporto qui le variazioni di torsione $\Delta \omega$ ottenute col filo $\mathrm{Fe}_{3}$ assoggettato al carico costante di g. 1100 ; i valori del campo alternato, in unità c. g. s., sono i seguenti :

$$
\begin{array}{lll}
\mathrm{H}_{1}=2,3 & \mathrm{H}_{3}=6,4 & \mathrm{H}_{3}=31,0 \\
\mathrm{H}_{4}=\tilde{\jmath} 3,8 & \mathrm{H}_{3}=78,0 & \mathrm{H}_{6}=91,5 .
\end{array}
$$

Mi limito a trascrivere le osservazioni, per campi crescenti e decrescenti, in un mezzo ciclo: nell' altra metà simmetrica $\mathrm{i}$ risultati non variano. 


\begin{tabular}{|c|c|c|}
\hline 0 & $\mathscr{\theta} \amalg \stackrel{m}{\rightarrow} \sigma \cdot \infty N$ & 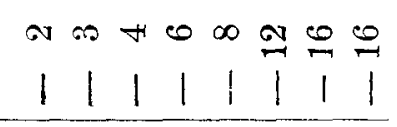 \\
\hline 5 & $\because 0100 \pi,-1$ & 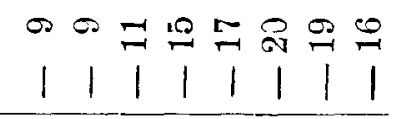 \\
\hline $\mathbf{I}^{*}$ & 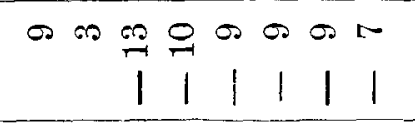 & 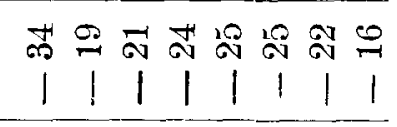 \\
\hline$x^{\infty}$ & 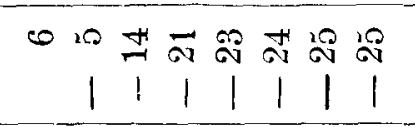 & 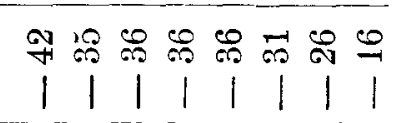 \\
\hline$\vec{F}$ & 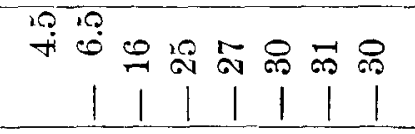 & 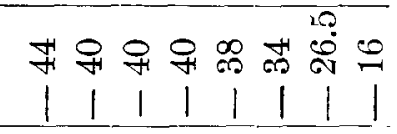 \\
\hline $\mathfrak{H}^{-5}$ & \# 111111 & 11111111 \\
\hline$z^{\circ}$ & 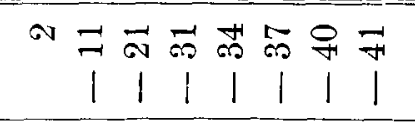 & 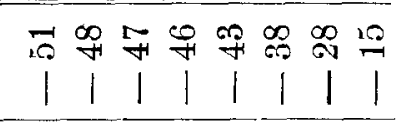 \\
\hline$\Xi ̊$ & 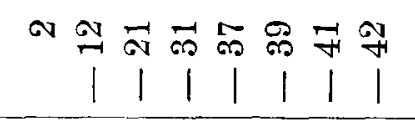 & $\begin{array}{c}1 \\
1\end{array}$ \\
\hline$\ddot{ت}$ & 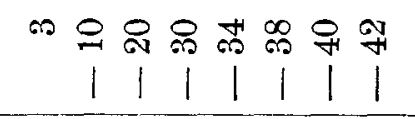 & $\begin{array}{cccccc}1 & 1 & 1 & 1 & 1 & 1\end{array}$ \\
\hline$\underbrace{-0}$ & 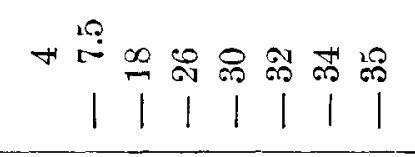 & 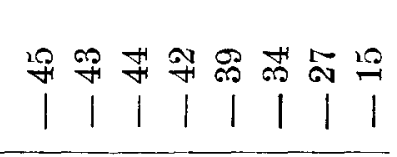 \\
\hline تే & $\begin{array}{c}\infty \\
0\end{array}$ & 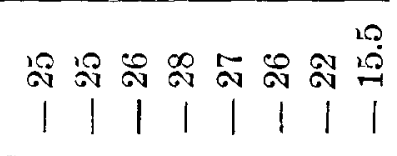 \\
\hline $\bar{a}$ & 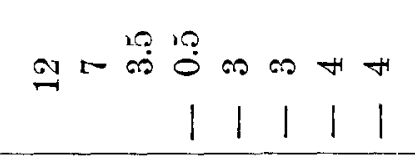 & 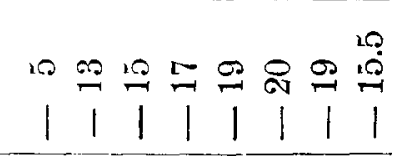 \\
\hline 3 & 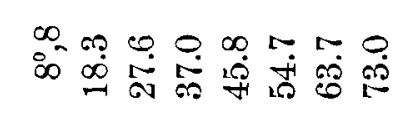 & 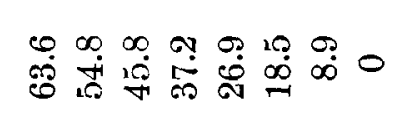 \\
\hline
\end{tabular}


Da qui si vede che:

Nella torsione: $a$ ) per piccole torsioni - dell' ordine di $0^{0}, 3$ per em. - il campo alternato $\mathrm{H}$ genera sempre aumenti di torsione $\omega$ che diventano sempre più piecoli quando $\mathrm{H}$ cresce, ma che al diminuire di $\mathrm{H}$ tornano a crescere, finchè a campo nullo resta un aumento permanente relativamente considerevole; $b$ ) per grandi torsioni - di $1^{0}, \bar{\partial}$ o più per cm. - la $\omega$ diminuisce sempre di più se $\mathrm{H}$ cresce, la diminuzione raggiungendo rapidamente un massimo col campo $\mathrm{H}_{5}$; al diminuire di $H$ le $\Delta \omega$ decrescono con rapidità, finchè cambiano di segno $\theta$ a campo nullo resta sempre un aumento di $\omega$, minore però più grande è $\omega ; c)$ per torsioni intermedie - da $0^{\circ}, 7$ a $1^{\circ}, 2$ per $\mathrm{em}$. - al crescer di $\mathrm{H}$ la $\omega \mathrm{da}$ prima aumenta, poi diminuisce e al decrescere di $\mathrm{H}$ si ha l' effetto inverso.

Nella detorsione : a) l' effetto del campo alternato è sempre quello di produrre una diminuzione di $\omega$ in ogni caso maggiore delle diminuzioni ottenute, per angoli $\omega$ uguali, nella torsione; b) al crescere di $\mathrm{H}$ le diminuzioni crescono, anche qui, rapidamente da prima, poi decrescono leggermente; esse aumentano pure man mano si detorce il filo fino ad un certo angolo oltre il quale - circa $1^{0}, \tilde{0}$ per cm. - decrescono; c) al diminuire di $\mathrm{H}$ le $\Delta \omega$, sempre negative, diventan sempre più piccole, ma a campo nullo resta ora una diminuzione residua che $s^{\prime}$ accresce quando $\omega$ impiccolisce; $d$ ) a torsione nulla il campo $\mathrm{H}$ più debole, provoca una diminuzione di $\omega$ che resta sensibilmente costante col variare di $\mathrm{H}$.

Altri fili esaminati in condizioni simili alle precedenti hanno dato risultati qualitativamente uguali a quelli trovati con $\mathrm{Fe}_{3}$.

Il comportamento del ferro in campi alternati risulta alquanto diverso da quello che manifesta in campi costanti, almeno finchè la torsione non supera un certo valore, avendosi allora nel primo caso degli aumenti di torsione che non si producono nell' altro. Inoltre nella detorsione si hanno ora delle $\Delta \omega$ sempre negative, ma superiori, a parità di $\omega$, a quelle avute nella torsione. 
Non semplice è l' indagine della causa o delle cause di tutti i risultati avuti, chè il filo di ferro è sede di fenomeni assai diversi fra loro. Oltre il fatto che la magnetizzazione percorre ora dei rapidi cicli è da considerare qui che il filo $\grave{e}$ per questo assoggettato a vibrazioni longitudinali e torsionali e per di più esso, pure in campo sì rapidamente variabile, subisce alterazioni di lunghezza il cui andamento generale sembra simile a quello che manifesta sotto l'azione di campi costanti ').

Riferendosi a quest' ultimo fatto è utile conoscere come variano gli effetti riscontrati al mutare del carico tensore, avendosi che, com'è noto, in campo costante coll' aumentare del peso traente diminuisce l'allungamento del ferro fino a scomparire del tutto; e poichè l' accorciamento che allora si produce è più cospicuo in campi più intensi, ho cercato di mettermi in queste condizioni aumentando l'intensità del campo alternato.

Del filo $\mathrm{Fe}_{6}$ che, esaminato nelle condizioni di $\mathrm{Fe}_{3}$ ha dato risultati simili a quelli già riportati, ho studiato il comportamento sotto tre carichi diversi e, lanciando la corrente alternata nella spirale esterna, ho generato i campi dei seguenti valori :

$$
\begin{array}{ll}
\mathrm{H}_{1}^{\prime}=69 & \mathrm{H}_{2}^{\prime}=138 \\
\mathrm{H}_{3}^{\prime}=207 & \mathrm{H}_{4}^{\prime}=271 \quad \text { unità c. g. s. }
\end{array}
$$

Le variazioni $\Delta \omega$ sono:

1) Austin. The Physical Review. Vol. X, 1900. 


\begin{tabular}{r|r|r|r|r|r|r|r|r}
\hline$\omega$ & $\mathrm{H}^{\prime}$ & $\mathrm{H}^{\prime}$ & $\mathrm{H}_{3}^{\prime}$ & $\mathrm{H}_{4}^{\prime}$ & $\mathrm{H}_{3}^{\prime}$ & $\mathrm{H}_{2}^{\prime}$ & $\mathrm{H}_{3}^{\prime}$ & 0 \\
\hline \hline 8.8 & -4 & -4 & -3 & -3 & -2 & -1 & -1 & 8 \\
17.0 & -15 & -17 & -17 & -19 & -17 & -15 & -14 & 6 \\
26.0 & -26 & -29 & -31 & -34 & -30 & -27 & -25 & 7 \\
36.0 & -37 & -40 & -41 & -45 & -40 & -38 & -35 & 3 \\
45.3 & -43 & -46 & -48 & -53 & -48 & -44 & -41 & 5 \\
55.5 & -51 & -56 & -59 & -64 & -57 & -53 & -49 & 2 \\
65.0 & -52 & -56 & -59 & -65 & -58 & -54 & -49 & 1 \\
& & & & & & & & \\
55.5 & -55 & -59 & -62 & -67 & -60 & -56 & -53 & -4 \\
45.4 & -50 & -54 & -56 & -60 & -55 & -52 & -48 & -2 \\
36.0 & -47 & -50 & -52 & -56 & -52 & -49 & -46 & -6 \\
26.2 & -41 & -43 & -45 & -48 & -45 & -43 & -40 & -11 \\
17.0 & -29 & -32 & -34 & -36 & -33 & -32 & -30 & -11 \\
8.7 & -20 & -21 & -21 & -22 & -21 & -20 & -20 & -5 \\
0 & -9 & -9 & -5 & -5 & -9 & -5 & -8 & -8
\end{tabular}

$\mathrm{Fe}_{6}$ g. 3000

\begin{tabular}{r|r|r|r|r||r|r|r|r}
\hline$\omega$ & $\mathrm{H}^{\prime}$ & $\mathrm{H}_{2}{ }_{2}$ & $\mathrm{H}^{\prime}{ }_{3}$ & $\mathrm{H}^{\prime}$ & $\mathrm{H}_{3}$ & $\mathrm{H}_{2}^{\prime}$ & $\mathrm{H}^{\prime}$ & 0 \\
\hline $9^{0} .0$ & -1 & -1.5 & -2 & -3 & -1 & -0.5 & -0.5 & 6 \\
17.9 & -10 & -11 & -12 & -13 & -11 & -9.5 & -9 & 5 \\
27.0 & -16 & -18 & -19 & -21 & -18 & -17 & -15.5 & 5 \\
36.2 & -24 & -26 & -28 & -30 & -27 & -25 & -23 & 5 \\
46.0 & -30 & -33 & -35 & -37 & -34 & -31 & -28 & 5 \\
55.8 & -35 & -38 & -40 & -44 & -40 & -37 & -33 & 4 \\
64.0 & -38 & -41 & -43 & -48 & -42 & -38 & -36 & 7 \\
& & & & & & & & \\
55.5 & -43 & -46 & -49 & -51 & -47 & -44 & -41 & -3 \\
45.7 & -38 & -40 & -43 & -45 & -41 & -40 & -36 & -1 \\
36.0 & -35 & -37 & -39 & -42 & -38 & -36 & -34 & -7 \\
26.7 & -28 & -30 & -32 & -33 & -30 & -28 & -27 & -6 \\
17.6 & -21 & -23 & -24 & -25 & -23 & -22 & -21 & -6 \\
8,9 & -13 & -13 & -14 & -15 & -13 & -12 & -12 & -6 \\
0 & -6 & -7 & -7 & -7 & -7 & -7 & -7 & -7
\end{tabular}


$\mathrm{Fe}_{6}$ g. 5000

\begin{tabular}{c|c|c|c|c||r|r|r|r}
\hline$\omega$ & $\mathrm{H}^{\prime}$ & $\mathrm{H}^{\prime}$ & $\mathrm{H}^{\prime}{ }_{3}$ & $\mathrm{H}^{\prime}$ & $\mathrm{H}^{\prime}$ & $\mathrm{H}_{2}^{\prime}$ & $\mathrm{H}^{\prime}$ & \multicolumn{1}{c}{0} \\
\hline \hline 17.0 & -8 & -8 & -8 & -11 & -8 & -7 & -6 & 6 \\
26.0 & -13 & -14 & -15 & -17 & -14 & -13 & -12 & 3 \\
35.5 & -17 & -19 & -20 & -22 & -20 & -18 & -16 & 4 \\
45.0 & -22 & -24 & -26 & -29 & -24 & -22 & -19 & 5 \\
54.8 & -25 & -28 & -30 & -33 & -28 & -26 & -23 & 10 \\
63.6 & -29 & -32 & -35 & -37 & -33 & -29 & -27 & 5 \\
& & & & & & & & \\
54.7 & -32 & -35 & -37 & -39 & -35 & -32 & -29 & -1 \\
45.5 & -29 & -31 & -33 & -36 & -32 & -30 & -27 & -2 \\
35.5 & -25 & -26 & -28 & -30 & -26 & -25 & -23 & -4 \\
26.0 & -21 & -22 & -23 & -25 & -22 & -21 & -19 & -4 \\
17.0 & -14 & -15 & -16 & -17 & -16 & -14 & -13 & -4 \\
8.3 & -10 & -11 & -11 & -12 & -10 & -9 & -9 & -6 \\
0 & -5 & -5 & -5 & -5 & -5 & -4 & -4 & -4
\end{tabular}

Si osserva principalmente che ora le $\Delta \omega$ sono sémpre negative, diminuiscono più cresce il carico tensore e aumentano sempre quando $\mathrm{H}$ cresce. Sembra dunque che applicando fin da principio un campo assai intenso l'aumento di torsione prima osservato in campi deboli non si produca; ciò, insieme al fatto che le $\Delta \omega$ crescon sempre maggiore diventa $H$, può anche far ritenere che il probabile accorciamento del filo sia la causa di queste diversità.

Ma poichó l'altro fatto, sempre costatato, del prodursi a coppia nulla una diminuzione di torsione per virtù del campo più debole che si mantiene poi all' incirca costante sembra indicare una netta azione di $\mathrm{H}$ sulla isteresi elastica, ho croduto opportuno di ricereare se questa azione si esercita in tutte le torsioni, tanto più che le vibrazioni suscitate dal campo variabile nei fili debbono pure avere una influenza presumibilmente non trascurabile.

A questo riguardo ho cercato da prima come si alterino le $\Delta \omega$ se si riduce la isteresi elastica con oscillazioni, decrescenti, intorno al valore di $\omega$ a cui si giunge. $\mathrm{Col}$ filo $\mathrm{Fe}_{\ddot{z}}$ 
ho proceduto in questo modo: prodotto un dato $\omega$, esamino l' azione di $\mathrm{H}$ crescente e decrescente, poi coll' estremità inferiore del filo-dinamometro compio delle oscillazioni intorno alla posizione da essa raggiunta che dal valore di $\pm 50^{\circ}$ si annullano gradatamente ed esamino di nuovo l' effetto di $\mathrm{H}$. I risultati sono $i$ seguenti, ove nella prima linea, per ogni valore di $\omega$, son segnate le $\Delta \omega$ ottenute nel primo caso, nell'altra quelle osservate nel secondo. 
VARIAZIONI DI TORSIONE

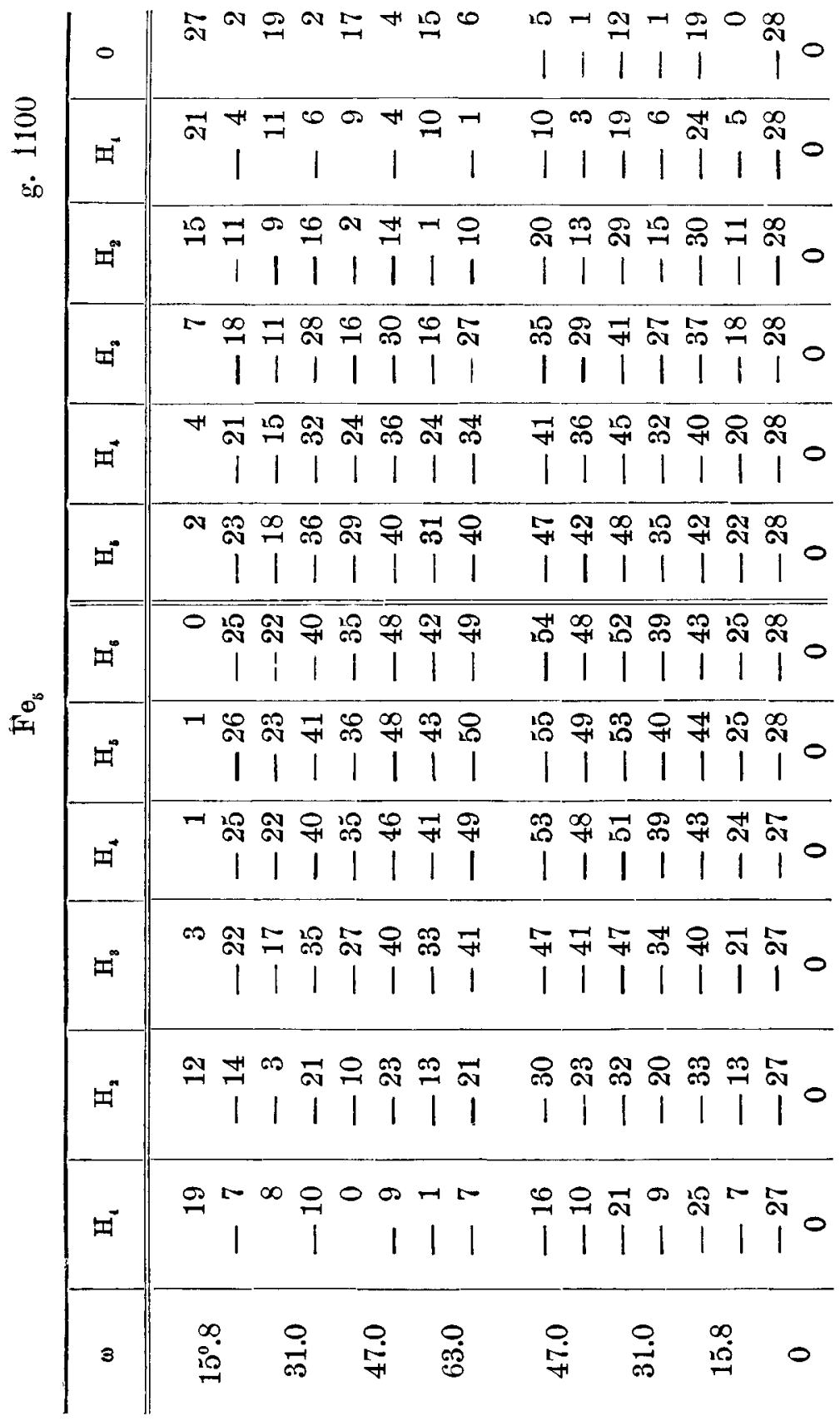


Si vede che per effetto delle oscillazioni torsionali decrescenti nella torsione scompaiono gli aumenti di $\omega$ per piccoli valori di questa, avendosi sempre $\Delta \omega$ negative; aumentano le $\Delta \omega$ negative e diminuiscono considerevolmente le $\Delta \omega$ residue che restano però sempre positive. E nella detorsione diminuiscono sempre le $\Delta \omega$ negative, più specialmente nei campi più deboli; diminuiscono, anche fino ad annullarsi, le $\Delta \omega$ residue, ed infine a coppia nulla si ha $\Delta \omega=0$.

Ora se facciamo le differenze $\Delta_{6}-\Delta_{1}$ fra le variazioni di torsione raggiunte sotto $H_{6}$ e quelle provocate coll' applicazione di $H_{1}$, e le altre $\Delta_{6}-\Delta_{0}$ fra le prime e le residue otteniamo :

\begin{tabular}{c|c||c}
\hline$\omega$ & $\Delta_{6}-\Delta_{1}$ & $\Delta_{6}-\Delta_{0}$ \\
\hline $15^{\circ} .8$ & -19 & -27 \\
& -18 & -27 \\
31.0 & -30 & -41 \\
& -30 & -42 \\
47.0 & -35 & -52 \\
63.0 & -37 & -52 \\
& -41 & -57 \\
47.0 & -38 & -55 \\
31.0 & -38 & -47 \\
& -31 & -40 \\
15.8 & -18 & -38 \\
& -30 & -24
\end{tabular}

Si nota subito la quasi perfetta coincidenza dei valori delle differenze, che si hanno prima e dopo le oscillazioni 
fatte subire al filo. Da questo si deduce immediatamente come tutta la diversità dei risultati che nei due casi si ottengono sia stabilita, fin dal principio, dal campo $\mathrm{H}_{1}$ più debole: l'azione del campo crescente da $\mathrm{H}_{1}$ in poi e quella di $\mathrm{H}$ decrescente dal massimo a zero restano sensibilmente le stesse, abbia o no il filo subito il trattamento elastico di torsione.

Inoltre si osserva pure che, a parità di $\omega$, i valori delle differenze calcolate sono molto prossimamente uguali nella torsione e nella detorsione. Ora, poichè nella detorsione di un filo non assoggettato ad altro trattamento elastico si hanno, in generale, per le $\Delta \omega$ valori assoluti maggiori che nella torsione, la cennata coincidenza dei valori delle differenze conferma il fatto che la diversità degli effetti è determinata principalmente dal campo più debole e che, riducendo con oscillazioni torsionali decrescenti la isteresi elastica del filo, le differenze fra le $\Delta \omega$ nella torsione e nella detorsione, provocate da campi $\mathrm{H}$ crescenti o decrescenti, tendono a scomparire.

Tutto ciò indica che l'effetto d'un campo alternato $\dot{\theta}$ duplice: esso annulla, o riduce considerevolmente, la isteresi elastica e diminuisce la torsione. E si comprende allora come l'effetto risultante che si ottiene da una prima applicazione del campo debba dipendere, oltre che dall' intensita di questo, anche dallo stato elastico del filo soggetto alla sua azione.

Era però da vedere se, data l'azione riduttrice d'isteresi prodotta dal campo alternato, l'applicazione successiva di esso potesse condurre a risultati che dei precedenti fossero la conferma e qui riferisco quelli avuti facendo agire $H$ per due volte di seguito: le successive sue applicazioni non producono più effetti sensibilmente diversi. 


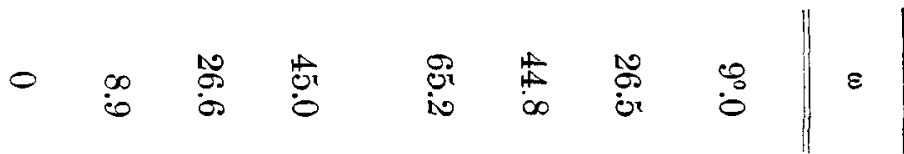

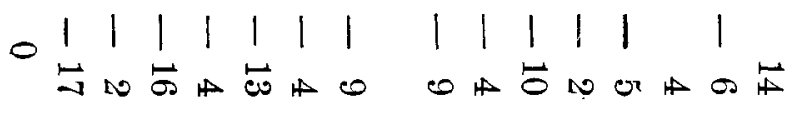

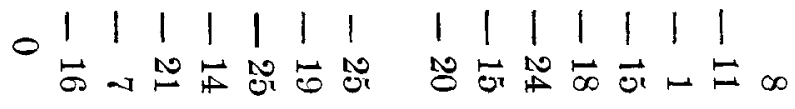

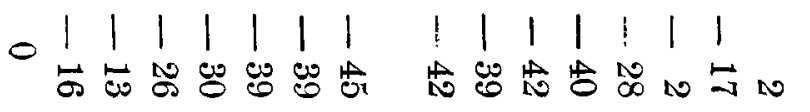

$\begin{array}{lllllllllllllll}0 & 1 & 1 & 1 & 1 & 1 & 1 & 1 & 1 & 1 & 1 & 1 & 1 & 1\end{array}$

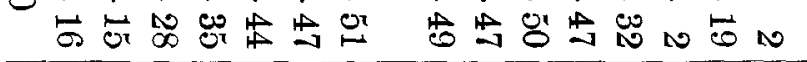

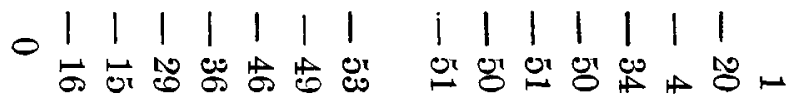

$\begin{array}{llllllllllllllll}0 & 1 & 1 & 1 & 1 & 1 & 1 & 1 & 1 & 1 & 1 & 1 & 1 & 1 & 1\end{array}$

-

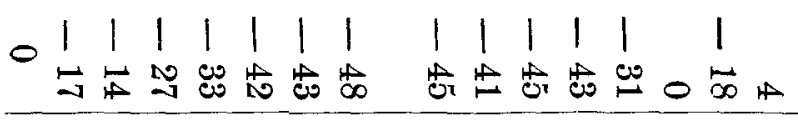

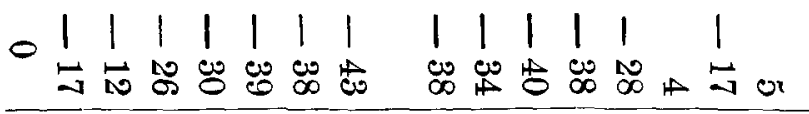

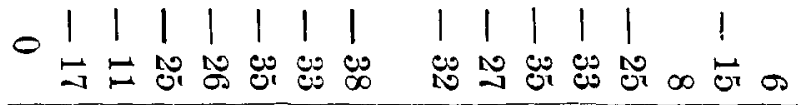

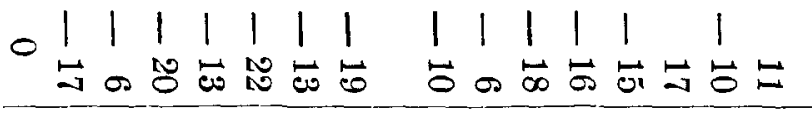

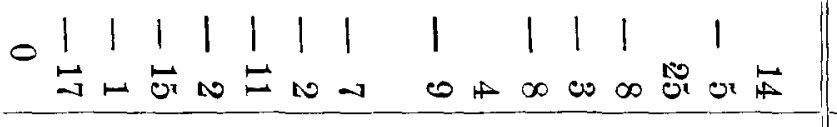

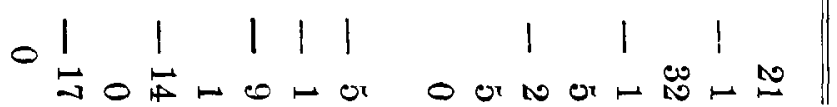


Essi ci mostrano che la seconda azione del campo consiste sempre nel produrre una diminuzione di torsione che decresce al diminuire del campo fino ad annullarsi, in generale, quando pur questo si annulla. Ritroviamo cosi ora dei risultati simili a quelli che si ebbero colle oscillazioni torsionali di $\mathrm{Fe}_{5}$.

Possiamo perciò concludere che l'azione iniziale d' un campo alternato crescente e poi decrescente fino a zero $\grave{e}$, principalmente, quella di ridurre la isteresi elastica nei fenomeni di torsione. Ciò che spiega come al ritorno a campo nullo si abbiano $\Delta \omega$ residue che sono positive nella torsione e come, col crescere del peso tensore, tutte le variazioni diventino sempre piu piccole, senza escludere che in quest' ultimo fatto possa pure influire sensibilmente il probabile effetto di magnetostrizione del ferro per virtù del campo alternato.

Considerando allora l' azione da questo prodotta nelle sue applicazioni successive, o dopo d'aver prodotto con forze esterne opportunamente applicate la riduzione $d$ 'isteresi elastica nel fenomeno di torsione, dobbiamo concludere che essa consiste sempre in una diminuzione dell' angolo di torsione $\omega$ crescente coll' ampiezza di $\omega$ e coll' intensità del campo e all' incirca dello stesso ordine di grandezza tanto se a valore di $\omega$ si giunge con valori crescenti o decrescenti di esso.

Possiamo allora arguire che anche una magnetizzazione rapidamente variabile può destare nel ferro delle forze interne non trascurabili ').

1) Qualunque sia l'intimo meccanismo di questo fenomeno, ho osservato che esso persiste anche quando, per effetto d'una deformazione permanente, si produce nel filo un grande scorrimento; chè applicando in tal caso il campo alternato, la velocità di rotazione dello specchio si altera notevolmente per riprendere il suo andamento all' annullarsi del campo.

Ho pure osservato che, specialmente nelle grandi torsioni e sotto piccoli carichi, l'applicazione e la soppressione brusca del campo più intenso generano delle lente ed assai ampie oscillazioni, talvolta multiple, della torsione del filo.

Sotto l'azione simultanea d' un campo alternato e d'uno costante, anche molto debole, le vibrazioni che prendono $i$ fili non permettono di eseguire le letture, quindi non ho potuto esaminare il comportamento della torsione quando il campo alternato, invece che intorno a zero, oscilla intorno a valori più o meno grandi. 


\section{Nichel.}

I fili di nichel che ho usati, sempre della lunghezia di $25 \mathrm{~cm}$, sono del diametro di $\mathrm{cm}$. 0,025 e ricotti con una corrente di 4 a. per $90 \mathrm{~s}$.

Sotto l'azione del campo alternato questi fili vibrano assai di più di quelli di ferro e non è sempre possibile eseguire le letture quando il peso che li tende è piccolo e la torsione è rilevante.

E per questo che ho dovuto aumentare fino a $500 \mathrm{~g}$. il carico tensore per avere risultati sicuri, che qui riferisco. 


\begin{tabular}{|c|c|c|}
\hline 0 & खृ & 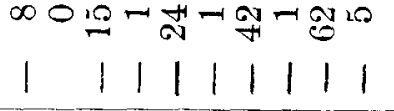 \\
\hline$ت$ & हु० & 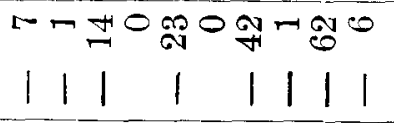 \\
\hline$\pi^{\prime \prime}$ & 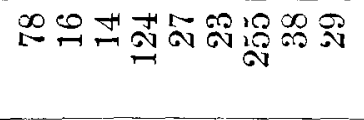 & 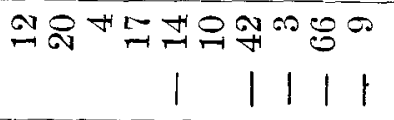 \\
\hline 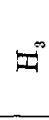 & 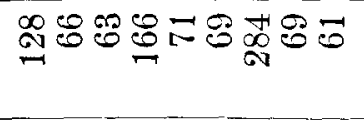 & 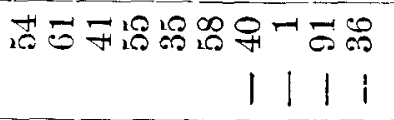 \\
\hline$\Psi^{*}$ & 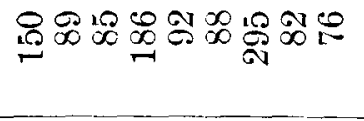 & 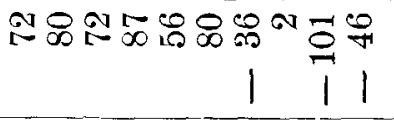 \\
\hline$\Psi^{-3}$ & 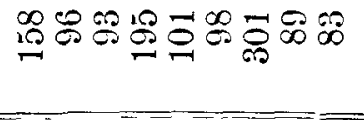 & 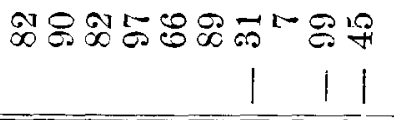 \\
\hline$\Psi^{0}$ & 10 & 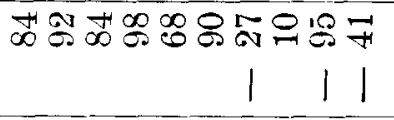 \\
\hline$Z^{20}$ & 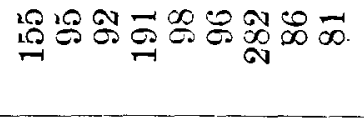 & $\begin{array}{r}1 \\
8 \pi \infty 71\end{array}$ \\
\hline $\overrightarrow{4}$ & 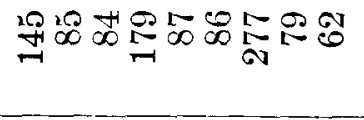 & 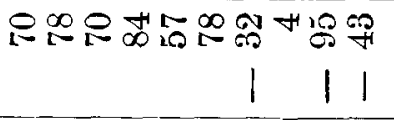 \\
\hline$\Psi^{\infty}$ & 원 & 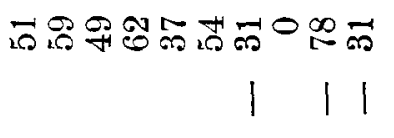 \\
\hline$\underbrace{-1}$ & बே & 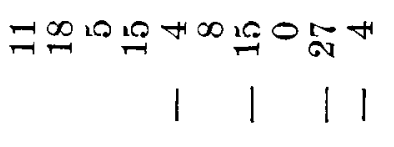 \\
\hline$\vec{E}$ & 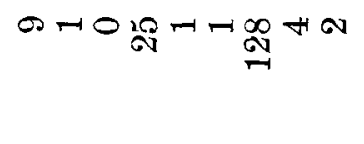 & $\begin{array}{l}1 \\
11\end{array}$ \\
\hline 3 & $\begin{array}{lll}1 & 0 & 0 \\
8 & 0 & 10 \\
0 & \infty\end{array}$ & 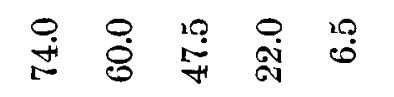 \\
\hline
\end{tabular}


Nella torsione la prima applicazione di $\mathrm{H}$ produce sempre variazioni di torsione positive considerevoli che crescono e diminuiscono con $\mathrm{H}$; ma all' azione successiva di questo si manifestano molto minori. Ciò indica che anche nel nichel il campo $H$ produce da prima una considerevole riduzione d'isteresi elastica e più cospicua maggiore $\dot{\theta} \omega$, come si scorge subito dall'esame delle $\Delta \omega$ residue a campo nullo; mentre l'azione ripetuta di $\mathrm{H}$ impiccolisce, sempre però di meno, tutte le $\Delta \omega$.

Nella detorsione, finchè $\omega$ è assai grande, coi campi più deboli si hanno $\Delta \omega$ negative, ma cogli altri valori di $H$ esse ritornano positive e crescenti coll'aumentare di $\mathrm{H}$. Una seconda applicazione di questo annulla le $\Delta \omega$ negative, accresce le positive, impiccolisce o annulla le residue.

Anche nella detorsione si manifesta chiaramente l'azione riduttrice $d$ 'isteresi del campo alternato; ciò potrà anche meglio vedersi nei risultati ottenuti col carico di $900 \mathrm{~g}$. 


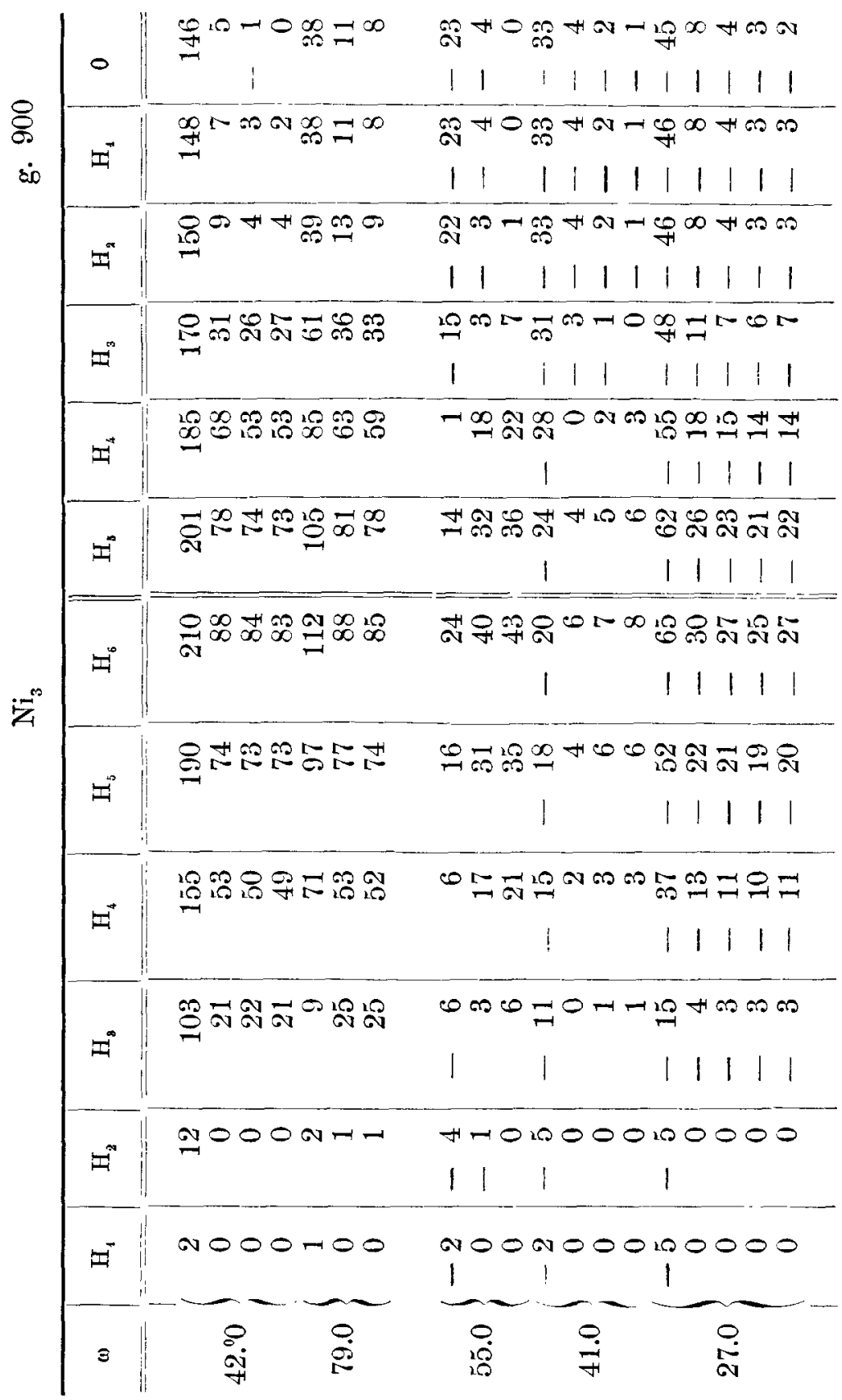


Intanto qui si osserva che nella torsione le applicazioni successive del eampo finiscono per produrre variazioni di torsione di valore praticamente costante.

La grande alterazione che $\mathrm{H}$ produce la prima volta viene considerevolmente diminuita se si fanno compiere al filo delle oscillazioni di torsione che dal valore massimo di $+30^{\circ}$ decrescono gradatamente: ciò appunto avvenne arrestandosi in ultimo alla torsione $\omega=79^{\circ}$. Ma si scorge allora, dal confronto dei risultati avuti con $\omega==42^{\circ}$, che nella riduzione d' isteresi è sempre più efficace l' azione di $\mathrm{H}$ di quella delle torsioni decrescenti, chè dopo queste $\mathrm{H}$ seguita a produrre una notevole diminuzione d'isteresi.

Nella detorsione, finchè col valore di $\omega$ non si ritorni ad angoli relativamente piccoli, la reiterata applicazione del campo converte le $\Delta \omega$ negative, da prima provocate, in positive, ma decrescenti man mano che $\omega$ impicciolisce e crescenti in valore assoluto quando $\mathrm{H}$ aumenta, almeno da un certo valore di questo in poi. Ma ritornati a torsioni assai piccole, le $\Delta \omega$ persistono sempre ad esser negative, nè, come ho riscontrato, cambiano di segno sottoponendo il filo a torsioni decrescenti.

Questo comportamento, che si ottiene anche in $\mathrm{Ni}_{3}$ con carichi minori e con tutti gli altri fili che ho saggiato, potrebbe far pensare che effettivamente il nichel presenti questa singolarità nella detorsione; ma potrebbe anche pensarsi che al ritorno a piccole torsioni il campo alternato, non riesca mai ad annullare completamente la isteresi elastica e che il residuo subisca una variazione temporanea sotto l'azione dei cambiamenti d' intensità di $\mathrm{H}$.

In favore di questa interpretazione stanno i risultati, che qui riporto, ottenuti con un filo $\mathrm{Ni}_{\mathrm{g}}$ crudo, in cui perciò l'isteresi elastica è molto più piccola che in quelli ricotti. 


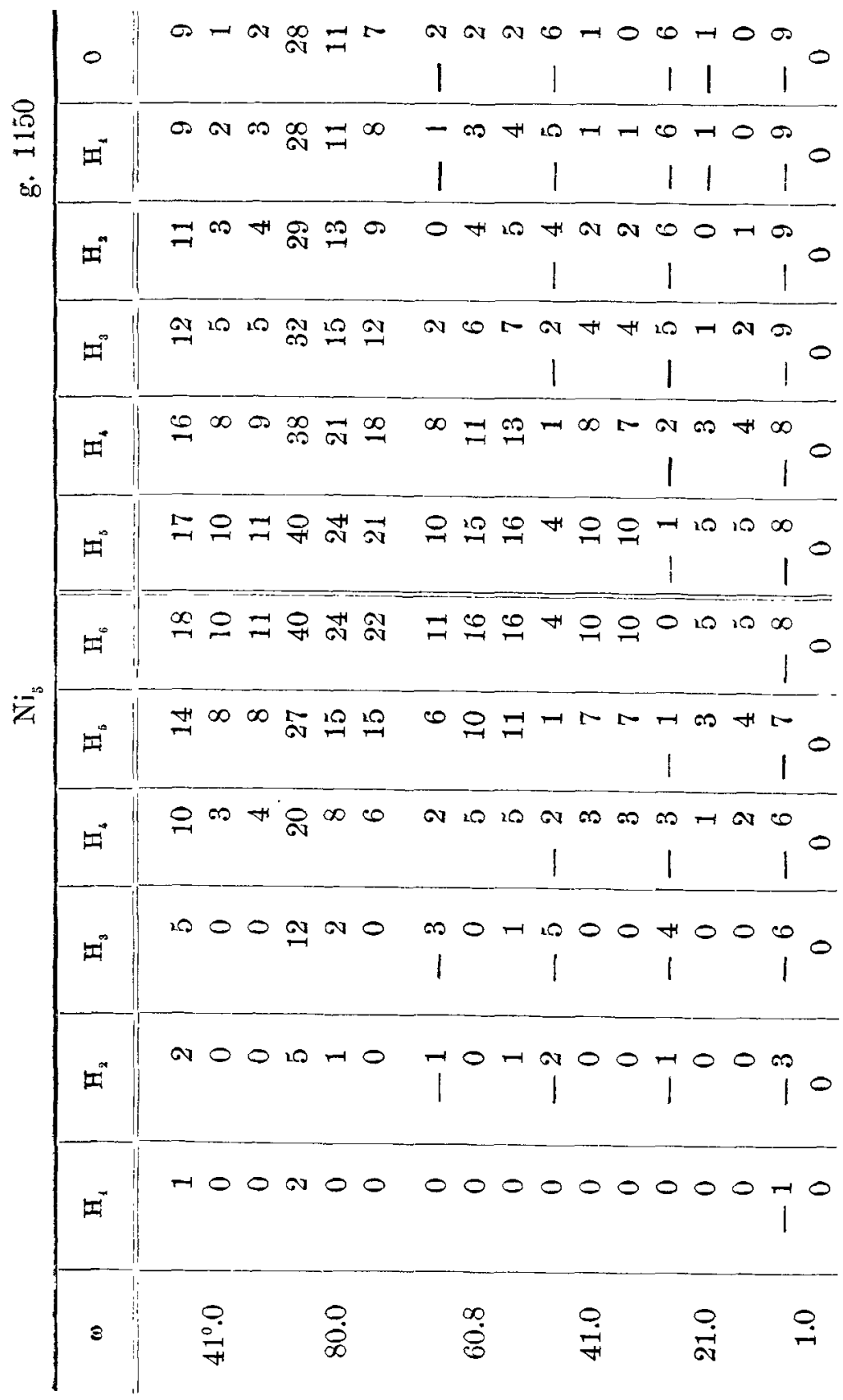


Mentre nella torsione il risultati song qualitativamente simili ai precedenti, nella detorsione si scorge subito che anche per piccoli valori di $\omega, l^{\prime}$ azione reiterata di $H$ produce sempre aumento di torsione, e a coppia nulla variazioni trascurabili.

Sembra quindi di poter ritenere che, ove l' isteresi elastica non sia troppo grande come nei fili ricotti, il primo effetto del campo è, durante tutta la detorsione, di ridurre quell'isteresi : ciò avvenuto, l'ulteriore applicazione di $\mathrm{H}$ manifesta nettamente l' azione di questo che consiste, come nella torsione, nel produrre un aumento dell' angolo di torsione $\omega$, dello stesso ordine di grandezza per uguali valori di $\omega$.

Se allora, riferendosi ai risultati offerti dai fili ricotti, si nota che le variazioni $\Delta \omega$ per l'azione ripetuta di $H$ sono sempre positive e si ammette l' esistenza d' una isteresi residua che possa subire delle variazioni in un certo accordo con quelle di $\mathrm{H}$, potrebbe forse ritenersi che le $\Delta \omega$ prodotte in principio della torsione debbano ascriversi, totalmente o in gran parte, a queste oscillazioni di isteresi; sebbene ricordando che nel ferro il campo alternato provoca sempre diminuzioni di torsione e che il nichel, anche in campi magnetici costanti, presenta in generale variazioni di segno contrario a quelle manifestate dal ferro, l'ipotesi sembra poco probabile.

D'altronde occorre pure osservare che la natura della isteresi stessa è diversa se ad un dato valore di $\omega$ si giunge con ampiezze crescenti o decrescenti della torsione ed inoltre l' effetto generale di $\mathrm{H}$ è nel primo caso, e fin da principio, quello di produrre una variazione persistente dello stesso segno di quella che si genera riducendosi l' isteresi : nel secondo caso invece gli effetti sono, fin dall' inizio, di segno contrario.

Conclusioni. - Dall' insieme dei risultati ottenuti nelle condizioni indicate sopra posson trarsi queste conclusioni generali.

Un campo magnetico alternato longitudinale altera sensibilmente la torsione di fili di ferro e di nichel: le variazioni prodotte suno crescenti e decrescenti coll' aumentare e diminuire dell' intensità del campo. 
L'azione di questo è duplice: produce cioè nei due metalli una riduzione di isteresi elastica accompagnata da una diminuzione di torsione nel ferro e da un aumento nel nichel.

In una prima applicazione del campo i due effetti si sovrappongono producendosi, specialmente nel ferro, fenomeni varî che dipendono dallo stato elastico dei fili; mentre nelle successive sue applicazioni si manifesta principalmente il secondo effetto.

Le variazioni che allora si hanno sono più grandi per angoli di torsione maggiori e dello stesso ordine di grandezza, tanto se ad una data ampiezza di torsione si giunge con valori crescenti o decrescenti di essa.

Il segno di queste variazioni è dunque lo stesso di quello che si ottiene col campo costante.

Napoli, gennaio 1913.

(Giunta il 15 gennaio). 